\title{
Editorial
}

\section{Next-generation adenoviral vectors: new and improved}

Traditional Ad vectors (E1 deleted) have a relatively large cloning capacity $(8 \mathrm{~kb})$, can be concentrated to high titers $\left(>10^{12}\right.$ particles $\left./ \mathrm{ml}\right)$, and can efficiently transduce a variety of tissues in vivo. Furthermore, Ad vectors can express transgenes episomally, and are not dependent upon integration or cell division for transgene expression to occur. Therefore, the risks of inadvertently activating endogenous oncogenes, and/or transmission through the germ line are minimized. In spite of episomal expression, (E1-) Ad vectors have been demonstrated to sustain high level expression of transduced genes for greater than 1 year in both immune-incompetent and immunecompetent animals. However, before their full clinical potential for long-term gene transfer may be realized, limitations of (E1-) Ad vectors must be addressed.

Current Ad vectors are constructed in a manner such that the E1 region of genes are replaced with a transgene flanked by appropriate promoter and polyadenylation elements. The recombinant (E1-) Ad vector genomes can be replicated (and subsequently encapsidated) only in human cells that express high levels of the E1 proteins in trans. In theory, after initial viral entry into any host cell lacking E1 activity, an (E1-) Ad vector should be replication defective, allowing expression only from the transgene to occur. In tissue culture systems, however, it is clear that (E1-) Ad are not completely replication defective, and at higher multiplicities of infection, the E2, E3 and $\mathrm{E} 4$ promoters are active and allow viral replication to proceed. ${ }^{1,2}$ As a result, (E1-) Ad vectors have been demonstrated to express the early genes, undergo genome replication, and express the L1 $\rightarrow 1.5$ structural proteins when utilized in vivo. Based upon these observations, a general hypothesis can be reached, namely that the isolation of modified Ad vectors, defective for expression of multiple Ad genes (in addition to the E1 genes) may be capable of improved performance characteristics in vivo, by preventing Ad vector genome-derived gene expression.

In response to the need to improve Ad vectors, two classes of Ad vector modification have generally been pursued. The former involves the isolation of so-called 'empty Ad' vectors that are devoid of most of the Ad genome, but are helper virus dependent for growth. The latter form of Ad vector modification results in the production of multiply deleted, helper virus-independent Ad vectors. A brief summary of the characteristics of these 'next-generation' Ad vectors is presented below.

\section{Helper virus-dependent Ad vectors}

It is well known that DNA viruses such as polyoma and SV-40 accumulate defective non-infective particles when serially propagated in tissue culture systems. Ad is no exception, and it was this propensity that allowed early investigations into viral packaging of Ad genomes to move forward. For example, when defective Ad particles were analyzed, the genomic content of the defective species varied, but they always contained the left hand portion of the viral genome, thereby localizing the Ad packaging signal.

A number of groups have capitalized upon defective genome packaging to create novel Ad vectoring systems, referred to here as helper-dependent (HD) Ad vectors. In most cases, HD Ad vector genomes are constructed by flanking a respective transgene cassette with the Ad inverted terminal repeat elements and the Ad packaging signals. This construct is then co-introduced into packaging cell lines with a replicating helper virus (usually an (E1-) Ad vector) and the two are serially propagated. The helper virus provides the $>80$ Ad protein functions required for Ad genome encapsidation. The final step of all HD Ad vector generation methods is the separation of the HD vector genome containing capsids from those containing the genome of the helper virus. Since the HD and helper virus capsids are identical, their separation is currently based upon their different buoyant densities, and is achieved via ultra-centrifugation-based techniques. Before buoyant density separation, some groups remove the packaging signal from the helper virus genome before encapsidation (an elegant maneuver facilitated by cre-lox-mediated recombination strategies) thereby significantly decreasing the amount of helper virus genomes that are eventually encapsidated. ${ }^{3}$

Multiple reports have demonstrated that HD Ad vectors can be produced to high titers, and they can have carrying capacities approaching $35 \mathrm{~kb}$. Several published reports have demonstrated the improved performance of HD Ad vectors in vivo. For example, HD Ad vector transduction of the human alpha-1-antritrypsin (hAAT) gene into the livers of C57BL/6 mice (tolerant of the hAAT gene) resulted in persistent and high level expression of hAAT, in contrast to the low level expression derived from transduction with an (E1-) AdhAAT vector. ${ }^{4}$ Furthermore, liver toxicity was significantly reduced with the use of the HD Ad vector. Similar results were obtained after HD Ad-mediated transduction of the murine leptin gene into tolerant (lean) mice, however leptin expression was transient when identical experiments were performed in $o b / o b$ mice. ${ }^{5}$ Despite transient leptin 
expression in $o b / o b$ mice, overall liver pathology was also significantly reduced with the use of the HD Ad vector. ${ }^{5}$ HD-Ad vector-mediated transduction of the bacterial $\beta$ galactosidase gene into the muscles of tolerant animals was associated with less inflammation and prolonged expression of the transgene, however, similar infections of non-tolerant animals resulted in transience of transgene expression and significant loss of the $\mathrm{HD} \mathrm{Ad}$ vector genome. $^{6}$

\section{Overall limitations}

Complete elimination of all Ad genes within the HD Ad vectors is an extreme benefit, and has certainly contributed to the improved toxicity profiles noted with the use of this class of vector in vivo. However, all HD Ad preparations described to date (including those incorporating packaging signal mutations in the helper virus genome) are contaminated with significant amounts of helper viruses. Since $>10^{11}$ Ad vector particles are usually injected into a mouse to achieve efficient transduction (obviously several log higher will be required for human use) even $0.1-1 \%$ contamination levels may adversely affect the potential of this vector class for use in some gene therapy situations. Some very small HD Ad genomes have also been found to be intrinsically unstable unless Ad encoded proteins are simultaneously present; other reports with HD Ad vectors have not demonstrated similar instabilities. Resolution of this paradox awaits further study. ${ }^{7}$ Finally, high level defective particle and/or replication-competent Ad (RCA) generation occurring during the serial propagation of HD and helper Ad vectors are documented problems that may be limiting, especially when considering large-scale HD Ad vector production for human use. ${ }^{8}$

\section{Helper virus-independent, multiply deleted Ad vectors}

In order to propagate a multiply deleted and helper virus-independent Ad vector, packaging cell lines must be developed that trans-complement the growth of the modified vector. Justification as to which of the 80 or more Ad genes to co-express in packaging cell lines would require an entire review of the Ad life cycle, and is therefore not provided in this discussion. Ad packaging cell lines co-expressing the E1 and E4 genes, the E1 and E2a genes, the E1, E4, and protein IX genes, and the E1 and pTP genes are just some of the combinations successfully isolated to date. In most instances, inherent toxicity of the respective Ad genes required the use of inducible promoters to prevent their co-expression until vector production was initiated. In contrast, Ad packaging cell lines co-expressing the E1 and polymerase genes, or the E1, polymerase, and pTP genes did not require inducible expression of the respective gene activities, demonstrating that not all Ad genes are inherently toxic when coexpressed. ${ }^{9,10}$ Most of the cell lines described above also allowed for high level production of the respectively deleted Ad vectors, although some cell lines were more efficient at vector production than others, likely due to toxicity issues associated with the coexpression of some Ad genes.

Successful isolation of this class of Ad vector (regardless of the location of the deletion) immediately imparts several benefits, including increased cloning capacities and helper virus independence. Since these vectors are autonomously replicating (in the appropriate cell line) large-scale production proceeds as for (E1-) Ad vectors, precluding dependence upon buoyant densitybased methods of purification. Furthermore, this class of vector has a theoretically decreased propensity to generate RCA, since multiple, independent reversion events at each of the deleted regions would be necessary to generate an RCA.

Investigations utilizing (E1-, E4-) Ad vectors has resulted in several important observations. Initial reports demonstrated long-term persistence of (E1-, E4-) Ad vectors encoding bacterial $\beta$-galactosidase either in nontolerant mice, or only in mice tolerant of bacterial $\beta$-galactosidase. ${ }^{11,12}$ However, subsequent reports demonstrated a significant limitation, namely that transgene expression can be extremely short-lived when utilizing an (E1-, E4-) Ad. Possibly, (E1-, E4-) Ad vectors lack an E4 encoded activity required to activate promoters typically utilized to drive transgene expression in Ad vectors. ${ }^{13,14}$ Resolution of the varying observations noted with the use of (E1-, E4-) Ad vectors therefore awaits further studies. (E1-, E2a-) Ad vectors have also been utilized in vivo, but hepatic transfer with (E1-) or (E1-, E2a-) Ad vectors encoding identical transgenes (bacterial $\beta$-galactosidase or hAAT) revealed no improvements with the use of the (E1-, E2a-) Ad vector. ${ }^{15}$ Similar results have been noted when null (encoding no transgene) multiply deleted Ad vectors were compared with null (E1-) Ad vectors. ${ }^{16}$ Recently, an (E1-, polymerase-) Ad vector encoding bacterial $\beta$-galactosidase was capable of persisting for greater than 2 months in immune-competent mice that were non-tolerant of the bacterial $\beta$-galactosidase transgene, in contrast to the rapid elimination noted with an identical (E1-) Ad vector. ${ }^{17}$ Reasons for the varied results are numerous, unfortunately, major differences in experimental design (such as location of the vector deletions, mouse strains utilized, transgenes utilized, and endpoints analyzed) preclude direct comparisons between these reports at this time.

\section{Overall limitations}

There are several limitations to the use of multiply deleted Ad vectors. Their high level production is extremely dependent upon the packaging cell line supplying the necessary trans-complementing gene functions, therefore if the cell lines are inadequate, vector production will be adversely affected. Furthermore, multiply deleted Ad vectors still retain significant portions of the Ad genome, which will likely negatively impact upon their overall toxicity profiles. How significant this impact will be is dependent upon several variables, including the location of the specific Ad vector deletions, the immunogenicity of the transgene encoded by the vector, and the specific gene therapy model being tested. Finally, deletion of subregions of the Ad genome can sometimes be associated with unforeseen problems (ie deletion of the E4 genes).

\section{Summary}

The ability of Ad vectors efficiently to transduce genes into a variety of tissues fuels the wide interest in optimizing this vector for potential use in human gene therapy protocols. The natural biology of the Ad allows for a var- 
iety of modification schemes to be tested. Inevitably comparisons between the vector classes described above will be made; these opinions should always be forwarded with caution, since the combination of multiple variables (inherent transgene immunogenicity, immune background of the host strain or species, antibody responses that may or may not be accompanied by CTL responses, doses of vector, promoter dysregulation, contamination with helper viruses, etc) may allow a modified vector to appear extremely useful in one set of experiments, and appear no different than an (E1-) Ad vector in another.

In all likelihood, the modifications described will also be combined, either among themselves or with other vectoring systems (so called 'hybrid-vectors') to improve the biological performance profiles of gene transfer vectors in general. Therefore, the improvements already noted with the use of 'next-generation' Ad vectors may only be the beginning of a trend, especially when one contemplates the number of modifications that can now be theoretically introduced into future 'new and improved' Ad vectors.

\section{A Amalfitano ${ }^{1,2}$}

${ }^{1}$ Department of Pediatrics, Division of Medical Genetics and ${ }^{2}$ Department of Genetics, Duke University Medical Center Box 2618 Medical Sciences Research Building Room 101B, Durham, NC 27710, USA

\section{References}

1 Nevins JR. Mechanism of activation of early viral transcription by the adenovirus E1a gene product. Cell 1981; 26: 213-220.

2 Amalfitano A et al. Production and characterization of improved Adenovirus vectors with the E1, E2b, and E3 genes deleted. J Virol 1998; 72: 926-933.

3 Parks RJ et al. A helper-dependent adenovirus vector system removal of helper virus by cre-mediated excision of the viral packaging signal. Proc Natl Acad Sci USA 1996; 93: 13565-13570.

4 Schiedner $\mathrm{G}$ et al. Genomic DNA transfer with a high-capacity adenovirus vector results in improved in vivo gene expression

5 Morsy MA et al. An adenoviral vector deleted for all viral coding sequences results in enhanced safety and extended expression of a leptin transgene. Proc Natl Acad Sci USA 1998; 95: 7866-7871.

$6 \mathrm{Chen} \mathrm{HH}$ et al. Persistence in muscle of an adenoviral vector that lacks all viral genes. Proc Natl Acad Sci USA 1997; 94: 1645-1650.

7 Lieber A, He CY, Kay MA. Adenoviral preterminal protein stabilizes mini-adenoviral genomes in vitro and in vivo. Nat Biotechnol 1997; 15: 1383-1387.

8 Hardy $\mathrm{S}$ et al. Construction of adenovirus vectors through crelox recombination. I Virol 1997; 71: 1842-1849.

9 Amalfitano A, Begy CR, Chamberlain JS. Improved adenovirus packaging cell lines to support the growth of replication-defective gene-delivery vectors. Proc Natl Acad Sci USA 1996; 93: 3352-3356.

10 Amalfitano A, Chamberlain JS. Isolation and characterization of packaging cell lines that coexpress the adenovirus E1, DNA polymerase, and preterminal proteins - implications for gene therapy. Gene Therapy 1997; 4: 258-263.

11 Wang $Q$ et al. Persistent transgene expression in mouse liver following in vivo gene transfer with a delta-E1/delta-E4 adenovirus vector. Gene Therapy 1997; 4: 393-400.

12 Gao GP, Yang YP, Wilson JM. Biology of adenovirus vectors with E1 and E4 deletions for liver-directed gene therapy. J Virol 1996; 70: 8934-8943.

13 Brough DE et al. Activation of transgene expression by early region 4 is responsible for a high level of persistent transgene expression from adenovirus vectors in vivo. J Virol 1997; 71: 9206-9213.

14 Dedieu JF et al. Long-term gene delivery into the livers of immunocompetent mice with E1/E4-defective adenoviruses. J Virol 1997; 71: 4626-4637.

15 Morral $\mathrm{N}$ et al. Immune responses to reporter proteins and high viral dose limit duration of expression with adenoviral vectors comparison of E2a wild type and E2a deleted vectors. Hum Gene Ther 1997; 8: 1275-1286.

16 Lusky $\mathrm{M}$ et al. In vitro and in vivo biology of recombinant adenovirus vectors with E1, E1/E2a, or E1/E4 deleted. J Virol 1998; 72: 2022-2032.

$17 \mathrm{Hu} \mathrm{H}$ Serra D, Amalfitano A. Persistence of an [E1-, polymerase-] adenovirus vector despite transduction of a neoantigen into immune-competent mice. Hum Gene Ther 1999; 10: 355-364. 